\title{
Evolving plastic responses to external and genetic environments
}

\author{
M. Reuter, M. F. Camus, M. S. Hill, F. Ruzicka and K. Fowler
}

Research Department of Genetics, Evolution and Environment, University College London, Gower Street, London WC1E 6BT, UK

\section{Key words}

phenotypic plasticity, sexual dimorphism, regulatory evolution

\begin{abstract}
Phenotypic plasticity can mitigate adaptive trade-offs in fluctuating environments but how plasticity arises is little known. New research documents this process in a bacterial system. We highlight remarkable parallels to the evolution of sexual dimorphism and argue that their approach can aid our understanding of adaptive conflicts between the sexes.
\end{abstract}

\section{Main text}

Virtually all organisms are subject to varying environmental conditions, be it seasonal differences in climate, different stages of the life cycle, or phases of population growth. These environmental fluctuations can create varying and sometimes conflicting demands on the phenotype. One way of accommodating these is to modulate the phenotype according to environmental conditions, a phenomenon known as phenotypic plasticity. A large body of literature has been dedicated to exploring the conditions under which plasticity is selectively favourable, and empirically documenting the extent to which plasticity occurs and has a heritable genetic basis (1).

What is less well known is how the gene regulatory networks that mediate phenotypic plasticity evolve in response to opposing selection pressures. A recent article by $\mathrm{Yi}$ and Dean now sheds some light on these questions (2). They used an experimental evolution approach to explore adaptation in Escherichia coli to a cyclical environment that alternated between two selection pressures, one for rapid growth and one for chemotaxis. These contrasting regimes pose opposing demands on the phenotype, generating a trade-off in energy allocation between cell division and motility. Using models of population growth as a function of division rate in the growth-environment and motility in the chemotaxisenvironment, $Y i$ and Dean were able to construct a fitness landscape on which to map the evolutionary trajectory of the experimental populations (3). They found that adaptation proceeded in three phases (Figure 1). In phase 1, populations moved quickly up the fitness gradient as they adapted to the specific experimental setup. In phase 2, further adaptation was constrained by the trade-off between growth and chemotaxis. Here, mutations that improved fitness in one of the environments decreased fitness in the other, with populations moving principally along the fitness isocline while changing minimally in overall fitness. Finally, the evolutionary constraint imposed by the trade-off was overcome in phase 3 , where the populations were able to occupy novel regions of the fitness landscape. 
Analysis of the evolving populations revealed that the trade-off between growth and chemotaxis was broken by the emergence of increased plasticity in motility, which was reduced during the logistic growth phase and increased in the stationary phase, when chemotaxis selection occurred. The authors were further able to determine the genetic basis of this novel plastic response, identifying a single non-synonymous substitution in FLiA - a transcription factor gene which plays a central role in the chemotaxis network - to be responsible.

Yi and Dean's results demonstrate how experimentally tractable microbial systems can be exploited to elucidate the adaptive solutions and genetic mechanisms by which trade-offs can be overcome. Integrating genetic work of this type with existing evolutionary theory and phenotypic or quantitative genetic data will clarify the relationship between the evolution of plasticity and its underlying molecular sensory and regulatory machinery.

However, the impact of this kind of research goes beyond understanding plastic responses to external conditions, and applies to other cases where plastic responses can accommodate opposing selection pressures-a point not made by $\mathrm{Yi}$ and Dean. One such instance is the evolution of sexual dimorphism (4). Akin to varying external conditions, the sexes constitute different genetic environments that impose divergent selection pressures and adaptive trade-offs on genes as they pass through males and females over successive generations $(5,6)$. These trade-offs constrain adaptation in a manner equivalent to $\mathrm{Yi}$ and Dean's phase 2, where populations are unable to simultaneously optimise both sex-specific phenotypes. Instead, they are expected to accumulate alleles with opposing effects on male and female fitness - i.e. 'sexually antagonistic' genetic variation (Figure 1). This evolutionary impasse can only be overcome by the emergence of molecular mechanisms, equivalent to the substitution in FLiA during Yi and Dean's phase 3. These enable the uncoupling of male and female phenotypes, leading to increased plasticity in sex-specific phenotypes, the resolution of sexual antagonism, and hence sexual dimorphism.

Exploiting parallels between external and sex-specific genetic environments will yield a better understanding of plastic phenotypes and the evolution of the genotype-phenotype map. The key questions to pose in this context include: how do adaptive constraints arise, what are their consequences for the genetic load and levels of standing genetic variation of populations, and finally, how are constraints resolved?

The presence of adaptive constraints implies that genetic variation for their resolution is neither readily available nor remains unavailable for long enough to lead to a state of detectable maladaptation. The factors limiting selectable variation will depend on the nature of the traits under selection and should, a priori, be similar in the case of environments or the sexes. If selection acts on the context-dependent expression levels of individual genes, then adaptation could be limited by epistatic interactions between regulatory mutations, as shown 
in elegant work on E. coli by Poelwijk et al. (7). Selection on coding variants might be even more constrained, as resolution most likely requires both gene duplication and regulatory divergence $(8,9)$.

The maintenance of genetic variation and the associated genetic load has been a major focus of research on sexual antagonism. Extending this to fluctuating environments would provide an interesting avenue of research, in particular if linked to molecular analysis of constraints and their eventual resolution. This would also allow to study the longer-term dynamics of antagonistic variation and emerging plasticity. One outstanding question here is whether the resolution of adaptive constraint allows for periods of rapid adaptation by increasing plasticity or whether populations evolve via repeating cycles of adaptive constraint and partial resolution (Fig. 1). Care is needed, however, when analysing genetic variation in response to environmental selection, because fluctuating environments can also favour the coexistence of fixed strategies, as diversifying bet-hedging strategies $(10,11)$, even where plasticity is not selectively favoured.

Finally, more data on the resolution of adaptive conflicts will provide insights into how genomes accommodate opposing selective demands on the phenotype, and whether these differ between responses to the environment and the sexes. The basic adaptive task is the same for environment- and sex-specific selection pressures: sensory information relating to each external or genetic environment needs to be converted into the expression of a specific set of gene products. Accordingly, one might expect plasticity and sexual dimorphism to rely on the same mechanisms to resolve adaptive conflicts, e.g. regulatory changes, gene duplication, or epigenetic effects. The mechanisms used in individual cases may depend predominantly on the genetic particularities of the traits under selection, but systematic differences between plasticity and dimorphism are also imaginable. For example, the predictability of fluctuations in selection pressures (higher in sexual than external environments) or the reliability with which the current environment can be sensed (again potentially higher with genetic or hormonal cues of sexual identity) will also influence which mechanisms can effectively resolve the conflict. In the long term, empirical data from studies like that by $\mathrm{Yi}$ and Dean and related theoretical work will help us establish the extent to which phenotypic plasticity and sexual dimorphism are analogous phenomena, as well as delineate the areas in which they diverge.

\section{References}

1. Pigliucci M. Phenotypic Plasticity: Beyond Nature and Nurture: Johns Hopkins University Press; 2001.

2. Yi X, Dean AM. Phenotypic plasticity as an adaptation to a functional trade-off. eLife. 2016;5:e19307.

3. $\quad$ Rockwood LL. Introduction to Population Ecology: Wiley-Blackwell; 2006.

4. Rice WR, Chippindale AK. Intersexual ontogenetic conflict. Journal of Evolutionary Biology. 2001;14(5):685-93. 
5. Bonduriansky R, Chenoweth SF. Intralocus sexual conflict. Trends in ecology \& evolution. 2009;24(5):280-8.

6. van Doorn GS. Intralocus sexual conflict. Annals of the New York Academy of Sciences. 2009;1168:52-71.

7. Poelwijk Frank J, de Vos Marjon GJ, Tans Sander J. Tradeoffs and Optimality in the Evolution of Gene Regulation. Cell. 2011;146(3):462-70.

8. Des Marais DL, Rausher MD. Escape from adaptive conflict after duplication in an anthocyanin pathway gene. Nature. 2008;454(7205):762-5.

9. Dalla S, Dobler S. Gene duplications circumvent trade-offs in enzyme function: Insect adaptation to toxic host plants. Evolution. 2016;70(12):2767-77.

10. Seger J, Brockmann HJ. What is bet-hedging? Oxford Surveys in Evolutionary Biology Oxford University Press. 1987;4:182-211.

11. Botero CA, Weissing FJ, Wright J, Rubenstein DR. Evolutionary tipping points in the capacity to adapt to environmental change. Proceedings of the National Academy of Sciences. 2015;112(1):184-9. 


\section{Figure legend}

Figure 1: Hypothetical evolutionary trajectory on an adaptive landscape across two environments, either external or genetic (i.e., sexes). Each dot represents the fitness distribution of a population at a given time-point. Blue contours represent fitness isoclines (lines of equal fitness), where darker shades equate to higher fitness. Populations evolve quickly up the fitness slope (phase 1) until they encounter fitness trade-offs (phase 2). Here, further fitness increases slow and we observe a build-up of genetic variance with opposing effects in the two environments (purple/blue). Further increases in mean fitness across environments are possible by the emergence of mutations that break the trade-off (phase 3). The trajectory of further evolution could either consist of repeated cycles of phases 1-3 or rapid adaptation following the successful uncoupling of fitness in the two environments. 


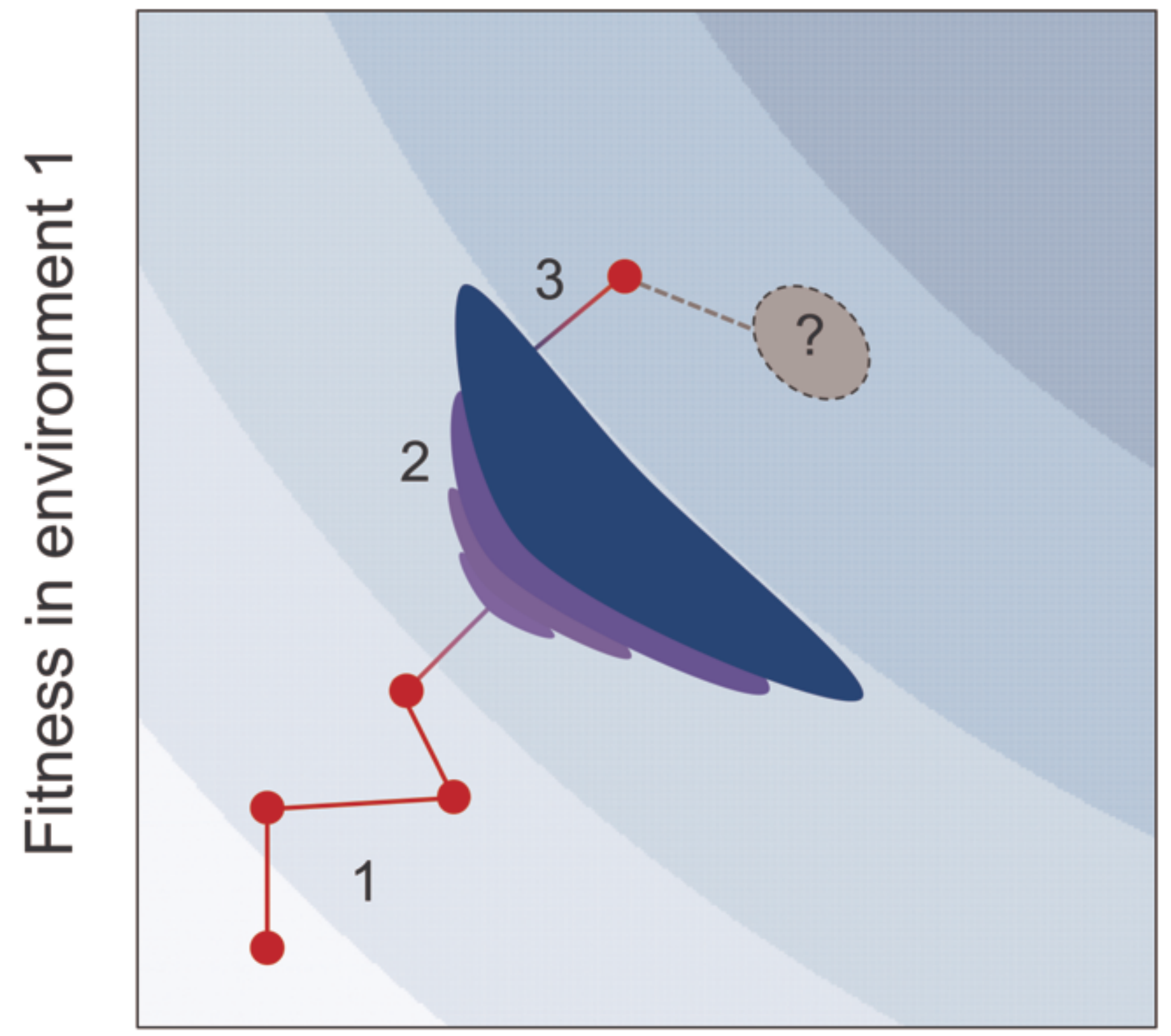

Fitness in environment 2 